AN. MED. INTERNA (Madrid) Vol. 19, N. $^{\circ} 7$, pp. $361-364,2002$

\title{
Gastroenteritis eosinofílica: espectro clínico de una misma entidad
}

\author{
C. DE LA SERNA HIGUERA, S. J. RODRÍGUEZ GÓMEZ, M. I. MARTÍN ARRIBAS, \\ J. MARTÍNEZ MORENO, A. PÉREZ VILLORIA
}

Unidad de Digestivo. Servicio de Medicina Interna. Hospital Virgen de la Concha. Zamora

\author{
EOSINOPHILIC GASTROENTERITIS: CLINICAL SPECTRUM OF \\ THE SAME DISEASE
}

\begin{abstract}
RESUMEN
La gastroenteritis eosinofílica es una entidad de etiopatogenia no aclarada y presentación excepcional. Sus síntomas clínicos son muy diversos, abarcando desde cuadros leves y de dolor abdominal inespecífico hasta episodios agudos de obstrucción intestinal que hacen preciso en ocasiones un tratamiento quirúrgico urgente. Este amplio abanico sintomático parece estar condicionado por el grado de infiltración eosinófila de la pared intestinal y el número de capas afectadas. Presentamos dos casos que ilustran la diversidad anatomo-clínica del cuadro y sus implicaciones terapéuticas.
\end{abstract}

PALABRAS CLAVE:Gastroenteritis esofçagica. Variantes clínicas.

\begin{abstract}
Eosinophilic gastroenteritis is a condition of unknown etiopathoge nesis and unusual description. Clinical symptoms are widely diverse ran ging from mild episodes of abdominal discomfort to acute intestinal obs truction which leads occasionally to urgent surgical approach. This wide range of clinical posisibilities seems to be secondary to the rate of eosi nophilic infiltration of the bowel wall and the number of layers involved. We report two cases showing that anatomo-clinical variety and their the rapeutic outcomes.
\end{abstract}

KEY WORDS: Eosinophilic gastroenteritis. Clinical variants.

De la Serna-Higuera C, Rodríguez-Gómez SJ, Martín-Arribas MI, Martínez-Moreno J, Pérez-Villoria A. Gastroenteritis eosinofílica: espectro clínico de una misma entidad. An Med Interna (Madrid) 2002; 19: 361-364.

\section{INTRODUCCIÓN}

La gastroenteritis eosinifílica (GEE) es una entidad de etiopatogenia desconocida y descripción infrecuente. Desde el primer caso descrito por Kaijser en 1937 (1), son menos de 300 los casos publicados en la literatura mundial (2), de los cuales 45 pertenecen a nuestro país (revisión bibliográfica MEDLINE 1966-1999). Las manifestaciones clínicas de la enfermedad y su evolución son muy diversas en función de la localización del área del tracto digestivo afecta y el grado de afectación eosinófila de las distintas capas de la pared intestinal. Todo ello ha contribuido a que la enfermedad, especialmente en sus formas leves haya permanecido infradiagnosticada hasta épocas recientes (3), apreciándose en los últimos años y particularmente en nuestro medio un número progresivamente creciente de nuevos casos probablemente basado en un mayor índice de sospecha clínica y en un estudio histológi- co dirigido. Presentamos dos nuevos casos que muestran la variabilidad clínica y evolutiva citada, así como los diversos abordajes terapeúticos posibles.

\section{CASOS APORTADOS}

Caso $n^{\circ} 1$ : Mujer de 32 años con antecedentes de alergia de contacto con metales y probable alergia a metamizol, que acude a Urgencias por un cuadro de dolor abdominal de localización hipogástrica con irradiación a ambos hipocondrios de 24 horas de evolución. En el momento del ingreso, el dolor aparecía focalizado en fosa ilíaca derecha, acompañándose de signos de peritonismo local. La analítica urgente reveló como único hallazgo leucocitosis (15.900) con neutrofilia (80\%) y recuento de eosinófilos normal. En la radiografía simple de abdomen se apreciaron múltiples niveles hidroaéreos de intestino delgado. Ante la sospecha de apendicitis aguda, se realizó laparotomía urgente, apreciándose abundante líquido libre intraabdominal y un segmento de ileon de $10 \mathrm{~cm}$ de aspecto inflama-

\footnotetext{
Trabajo aceptado: 11 de enero de 2001
}

Correspondencia: Carlos de la Serna Higuera. Unidad de Digestivo. Servicio de Medicina Interna. Hospital Virgen de la Concha. Avenida de Requejo 31-33. 49022 Zamora. 
torio con engrosamiento de paredes y equimosis en el meso, que se resecó selectivamente. El informe anatomo-patológico describía una pared de intestino delgado con intenso infiltrado inflamatorio eosinofílico que afectaba a serosa, muscular y submucosa, estando ésta última muy edematosa con abundantes microabscesos eosinofílicos. La mucosa aparecía respetada y no se apreciaban signos de afectación en la grasa mesentérica. El líquido ascítico era de características exudativas (proteínas: $5 \mathrm{~g} / \mathrm{dl}$ ) con recuento eosinófilo elevado $(5.800 / \mathrm{ml})$. Los cultivos resultaron negativos. La paciente permaneció asintomática durante ocho meses, reingresando por un nuevo episodio de dolor abdominal cólico de cuatro días de evolución localizado en hipogastrio y con irradiación a ambas fosas renales. Presentaba ritmo intestinal normal, sin naúseas ni vómitos. Al ingreso se objetivó fiebre de $38^{\circ}$ y dolor difuso a la palpación profunda abdominal sin signos de peritonismo local. La analítica presentaba únicamente leucocitosis $(18.100)$ y neutrofilia $(75 \%)$. Eosinófilos normales. Radiografía de abdomen sin hallazgos patológicos. En la ecografía abdominal se apreciaba una pequeña cantidad de líquido libre perihepático y en gotieras paracólicas, con un asa de pared engrosada y edematosa en fosa ilíaca derecha sugerente de proceso inflamatorio local. Se realizó un tránsito gastrointestinal que mostraba una estenosis parcial de aproximadamente $15 \mathrm{~cm}$ a nivel de ileon que permitía el paso de contraste. El estudio posterior reveló cifras de IgE elevadas $(368 \mathrm{ng} / \mathrm{dl}$ al ingreso -normal hasta $85-$ y $2.000 \mathrm{ng} / \mathrm{dl} \mathrm{diez}$ días después). Tests cutáneos a inhalantes y alimentos -RAST a proteínas de huevo, leche, pescado, trigo y cerdo- negativos. VSG, factor reumatoide, autoanticuerpos, serología hidatídica y para Anisakis Simplex, coprocultivos, determinación de huevos y parásitos en heces, negativos o normales. Se realizó una gastroscopia sin apreciarse hallazgos patológicos, tomándose biopsias múltiples de esófago, estómago y duodeno en las que no se detectaron infiltrados eosinófilos.

Ante la sospecha de un nuevo brote de enteritis eosinofílica, se inició tratamiento con prednisona a dosis de $40 \mathrm{mg} /$ día, comenzando mejoría clínica evidente a las 48 horas. Cinco días después de iniciado el tratamiento, la paciente se encontraba completamente asintomática. Los controles clínico-analíticos realizados a los tres y doce meses del alta, resultaron normales en todos sus parámetros.

Caso $n^{\circ}$ 2: Mujer de 26 años, sin antecedentes de interés ni alergias conocidas, remitida a consulta por cuadro de dos meses de evolución de dolor abdominal episódico autolimitado, sin relación con la ingesta, localizado preferentemente en región periumbilical, con irradiación ocasional a fosa ilíaca izquierda, asociado ocasionalmente a episodios de diarrea líquida de 3-4 deposiciones diarias, sin productos patológicos. No presentaba otra sintomatología digestiva asociada. Había recibido tratamiento sintomático con espasmolíticos, sin experimentar clara mejoría clínica. La exploración física reveló únicamente dolor selectivo a la palpación profunda en fosa ilíca izquierda, sin signos locales de irritación peritoneal. La analítica presentaba valores normales en todos sus parámetros, incluyendo recuento leucocitario, neutrófilos, eosinófilos y VSG. Se realizó una ecografía abdominal que resultó normal. En la colonoscopia, se apreciaba un ileon terminal con mucosa difusamente eritematosa, con una pequeña lesión de aspecto ulcerativo fibrinado de aproximadamente 3 $\mathrm{mm}$. Se tomaron biopsias múltiples en las que se apreciaron signos inflamatorios inespecíficos con un denso infiltrado eosinófilo difuso sin presencia de granulomas locales o abscesos crípticos. Las determinaciones complementarias de IgE, factor reumatoide, autoanticuerpos, serología hidatídica y para Anisakis Simplex, coprocultivos y determinación de huevos y parásitos en heces resultaron normales o negativas. El tránsito intestinal presentaba irregularidad marcada de la mucosa a nivel de ileon terminal, sugerente de proceso inflamatorio local, sin imágenes de estenosis. La gastroscopia resultó normal, tomándose biopsias a distintos niveles, apreciándose únicamente agregados eosinófilos en mucosa de bulbo y segunda porción duodenal, así como en antro gástrico, con hallazgos locales compati bles con gastritis crónica superficial. Se realizó seguimiento clínico, encontrándose la paciente asintomática en el momento de concluir el estudio. Esta situación se mantuvo en revisiones posteriores a los $3 \mathrm{y}$ 10 meses, por lo que no se planteó el inicio de tratamiento específico corticoideo.

\section{DISCUSIÓN}

La gastroenteritis eosinofílica (GEE) es una entidad que ha permanecido probablemente infradiagnosticada hasta épocas recientes $(2,3)$ desde su primera descripción en 1937 (1). Este hecho viene avalado por la cantidad creciente de casos descritos en las últimas dos décadas, cifra que prácticamente duplica el número de publicaciones en el amplio intervalo anterior (2). La bibliografía española tampoco es ajena a esta tendencia, y aun tratándose de una entidad infrecuente, se aprecia un progresivo incremento de los casos notificados desde el primero publicado en 1973 (4). Esta tendencia se hace especialmente notoria en los últimos cuatro años (5-14) y en determinadas formas localizadas de la enfermedad (14). Todo ello pudiera estar determinado por un mayor índice de sospecha de esta enfermedad que ha llevado a diagnosticar casos con clínica inespecífica o sintomatología localizada que antes pasaban desapercibidos, mas aún considerando las remisiones espontáneas ocasionales que se describen con frecuencia15,como en el segundo de los casos aportados. En todo caso, se trata de una entidad clínico-patológica muy infrecuente y de expresión sintomática muy diversa, testimonio de su variada localización anatómica y grado de afectación histológica. El infiltrado eosinofílico parietal es la característica específica que define a la enfermedad: este infiltrado debe ser selectivo del tubo digestivo -descartándose una posible participación local en el contexto de un síndrome hipereosinofílico sistémico- e idiopático -fuera del contexto de determinadas enfermedades parasitarias, alérgicas, autoinmunes o humorales- $(15,16)$. Los mecanismos etiopatogénicos subyacentes no están completamente aclarados: se han implicado reacciones de hipersensibilidad inmediata tipo I ante determinados alergenos $(5,8)$-aunque éstos no están presentes en más de la mitad de los casos- (3) y otros mediados por IgE de presentación aún más excepcional (17). Ambas características están presentes en el primer caso de los descritos, en el que se asocia una afectación de la serosa, hecho excepcional en las formas con IgE elevada y antecedentes alérgicos (18). La eosinofilia sanguínea, aun siendo el dato analítico más frecuente, no constituye un criterio diagnóstico por si mismo, al poder estar ausente en porcentajes variables, que en algunas series se aproximan al 90\% de los casos (19). Los casos con afectación extensa, de predominio seroso presentan con mayor frecuencia ascitis, hipereosinofilia3 y mayores recuentos absolutos de eosinófilos en líquido ascítico (2) aunque en ocasiones se pueden hallar cifras normales a este nivel (20). Es característica la VSG normal o ligeramente elevada2.

El espectro clínico de la enfermedad es muy amplio, en relación con la localización del tramo afecto -puede existir afectación a cualquier nivel desde esófago hasta colon, y ocasionalmente abarcar de forma continua todo el tubo digestivo(21) y con la profundidad del infiltrado mural eosinófilo. En las series más largas publicadas (15) y también en nuestro país (3), el estómago y el intestino delgado fueron los segmentos más frecuentemente afectados, aunque quizá el dato pudiera estar sesgado por la menor tendencia a biopsiar de forma ruti- 
naria a otros niveles y más aún teniendo en cuenta la ausencia de imágenes endoscópicas específicas (2). El grado de infiltrado eosinófilo de la pared intestinal condiciona las tres formas clínicas definidas por Klein y cols. (18) según la capa predominantemente afecta. Las formas mucosas se caracterizan por dolor abdominal, diarrea y pérdida de peso que en ocasiones pueden dar lugar a un síndrome malabsortivo: se trata de los casos más frecuentes y más vinculados a antecedentes de alergia o atopia (hasta en el $50 \%$ de los pacientes) (15). Las formas con afectación predominante de la muscular se asocian con cuadros de obstrucción intestinal como consecuencia del engrosamiento del tramo afecto, fundamentalmente a nivel gástrico y de intestino delgado $(15,18,19)$ y mucho más excepcionalmente en colon (22). Estas presentaciones clínicas justifican los hallazgos radiológicos más frecuentemente asociados al proceso, como son el engrosamiento de pliegues y la estenosis (23). La tercera forma clínica, la menos frecuente, presenta afectación predominante de la serosa, asociándose de típicamente con la presencia de ascitis eosinofílica secundaria a la inflamación local que afecta también al peritoneo visceral adyacente (18). En estos casos, el inicio sintomático es ocasionalmente, en forma de abdomen agudo quirúrgico $(10,12)$. Estas formas serosas presentan de forma casi constante una afectación asociada transmural, siendo excepcionales los casos en los que la mucosa está indemne (24), como en el primero de los casos aportados.

Sin embargo, esta clasificación esquemática es en cierta medida arbitraria, dado que se ha comprobado que la infiltración eosinófila afecta siempre, en mayor o menor grado a todas las capas de la pared, siendo los síntomas los propios de aquella en que la afectación es predominante. Esto se pone en evidencia en los casos en los que se dispone de piezas de resección quirúrgica, o en aquellos otros en los que informando la biopsia endoscópica de afectación selectiva de la mucosa, la presencia de ascitis hace suponer una afectación simultánea de la serosa (3). Los dos pacientes que se presentan, ilustran esta variedad clínica, desde la afectación mucosa selectiva que justifica un cuadro de diarrea y dolor abdominal inespecífico, hasta la afectación transmural que dio lugar a un episodio de abdomen agudo y obstrucción intestinal con presencia de ascitis eosinofílica.

El diagnóstico implica por tanto un alto índice de sospecha clínica, así como la demostración histológica del infiltrado eosinófilo. Sin embargo, dada la distribución parcheada de la enfermedad así como la posible presencia de formas sin infiltración mucosa, pueden producirse frecuentes falsos negativos en las tomas biópsicas. Por ello en el diagnóstico endoscópico de la enfermedad, se recomienda la realización de biopsias múltiples (mínimo de seis) (2), a distintos niveles, para lograr un diagnóstico de extensión de la enfermedad. Se debe considerar siempre la mayor rentabilidad diagnóstica de las tomas a nivel de antro gástrico y duodeno y los posibles falsos positivos de las tomas a nivel de tercio distal esofágico en pacientes con reflujo gastro-esofágico -principal causa de eosinofilia a este nivel- (25) o esofagitis de otra etiología. Se deben de tomar muestras también de las zonas de aspecto endoscópico normal, dado que las alteraciones mucosas son inespecíficas y poco frecuentes (en menos del $20 \%$ de los casos con afectación duodenal en algunas series) (3). Igualmente se han de realizar de forma repetida en pacientes con eosinofilia periférica, clínica gastrointestinal de origen incierto y biopsias previas negativas. En el caso de resultar precisa la valoración de la posible afectación transmural, el abordaje por PAAF guiada por ecografía o TAC puede evitar las biopsias transmurales intraoperatorias recomendadas inicialmente (7. Actualmente la laparoscopia constituye un procedimiento alternativo eficaz en los casos de diagnóstico difícil no accesibles por otras técnicas $(26,27)$.

En el diagnóstico diferencial se debe considerar la existencia de otros cuadros clínicos que cursan no solo con eosinofilia periférica, sino ocasionalmente con infiltrados eosinófilos del tubo digestivo que pudieran condicionar sintomatología inespecífica local (2): infestaciones parasitarias, alergias farmacológicas -básicamente a sales de oro,azatioprina,clormetiazol y carbamacepina-, afectación intestinal por enfermedades reumatológicas, mastocitosis sistémica, linfomas y adenocarcinomas, enfermedad inflamatoria intestinal, sindrome hipereosinofílico primario y pólipo fibroide inflamatorio.

El tratamiento abarca distintas opciones condicionadas por la sintomatología predominante, que a su vez deriva del distinto grado de afectación mural. En aquellos casos en que la sintomatología es leve y de presentación episódica está justificada la actitud conservadora con abstención terapéutica $(2,15)$ seguimiento clínico y tratamiento sintomático si fuera preciso. En los pacientes con afectación selectiva de la mucosa y sin evidencia clínica de infiltración de muscular o serosa se ha recomendado la realización de tratamiento dietético, especialmente si existen antecedentes de hipersensibilidad alimentaria (17); en todo caso parece que la mejoría clínica es poco frecuente, y de producirse, transitoria (2) incluso en aquellos pacientes con antecedentes alérgicos en la anamnesis. En el extremo opuesto del abanico terapéutico, el abordaje quirúrgico se debe evitar en todo caso, salvo cuando se presentan complicaciones evolutivas (obstrucción, perforación), sospecha de cáncer o refractariedad al tratamiento esteroideo, en cuyo caso, el propio diagnóstico de GEE quedaría en entredicho. Sin embargo, como en el primero de los casos referidos, con cierta frecuencia es la laparotomía la que lleva al diagnóstico inicial de la enfermedad, en un paciente que acude por un cuadro obstructivo o de abdomen agudo $(10,12,22)$. En estas ocasiones, la resección del segmento afectado no es garantía de un mejor curso evolutivo dado que hasta en el $67 \%$ de los casos en las series más largas (19), la enfermedad recidiva posteriormente.

En la actualidad, los mecanismos inmunoalérgicos implicados en la patogenia de la enfermedad, hacen que el empleo de corticoides y -ocasionalmente- inmunosupresores constituyan la base de la terapéutica. Inicialmente la asociación frecuente de antecedentes atópicos así como la presencia de mastocitos activados llevó al empleo de antihistamínicos como el ketotifeno (28) sin resultados concluyentes posteriores. Mejores resultados se han obtenido con el empleo del cromoglicato disódico, que actúa no sólo previniendo la degranulación mastocitaria, sino también reduciendo la absorción de antígenos del intestino delgado. Aunque los resultados son discretos, se recomienda su empleo sólo en el caso de ausencia de respuesta o refractariedad al tratamiento corticoideo o si este no estuviera indicado $(11,29)$. La dosis exacta no está bien definida; sin embargo para obtener respuesta sostenida con buena tolerancia, se recomiendan $200 \mathrm{mg}$ al día repartidos en cuatro tomas (2).

En la actualidad los corticoides constituyen el tratamiento de primera elección con excelentes resultados y tolerancia, especialmente en las formas con afectación serosa (15) y mus- 
cular en las que se consigue la reversión de los cuadros obstructivos en pacientes previamente diagnosticados, haciendo innecesario el tratamiento quirúrgico. Las dosis habituales empleadas oscilan entre los 20-40 mg en adultos, obteniéndose una rápida respuesta que confirma el diagnóstico y permite una progresiva disminución de la dosis tras siete días de tratamiento, hasta su suspensión definitiva, aunque en ocasiones la recidiva clínica obliga a establecer un tratamiento de mantenimiento a dosis bajas. En los casos de corticodependencia puede ser preciso añadir azatioprina para reducir la dosis total necesaria de corticoides. Sin embargo no se dispone de series largas que permitan conocer su efectividad real (2).

El pronóstico a largo plazo de la enfermedad es favorable, con un curso en ocasiones recidivante que precisa de sucesivos ciclos de tratamiento corticoideo. No se ha descrito la aparición de secuelas a largo plazo, aumento del riego de desarrollo de cáncer gastrointestinal o disminución de la expectativa de vida. Sin embargo, el escaso número de pacientes y la ausencia de seguimientos a largo plazo impiden extraer conclusiones definitivas a este respecto.

\section{Bibliografía}

1. Kaijser R. Zur kenntuis der allergischen affektioner desima verdanungskanal von standpunk desima chirurgen aus. Arch Klin Chir 1937; 188: 36-64.

2. Talley NJ. Eosinophilic gastroenteritis. En: Feldman M, Schardschmid BF, Sleisenger MH, eds. Sleissenger and Fortran's Gastrointestinal and Liver Disease, 6th. de. Philadelphia, WB Saubders, 1998: 1679-1688.

3. Ruiz-Montes F, Reñé JM, Rubio M. Gastroenteritis eosinofílica: Revisión de los casos publicados en España y comparación con la literatura extranjera. Rev Esp Enf Ap Digest 1992; 81: 270-279.

4. Vázquez JJ, Soleto E, Sánchez J. Pancreatitis and eosinophilic gastroenteritis. Int Surg 1973; 58: 415.

5. Gómez B, Tabar AI, Tunon T, Larrinaga B, Alvarez MJ, García BE Olaguibel JM. Eosinophilic gastroenteritis and Anisakis. Allergy 1998; 53: $1148-1154$

6. Martí Carbonell J, Giro JM, Terradas F, pons-Porta A. Gastroenteritis eosinofílica de presentación atípica en un adolescente. An Med Interna (Madrid) 1998; 15 (11) 615-616.

7. Marco-Domenech SF, Gil-Sánchez S, Jornet-Fayos J, Ambit-Capdevila S, González-Anon M. Eosinophilic gastroenteritis: percutaneous biopsy under ultrasound guidance. Abdom Imaging. 1998; 23 (3): 286-288.

8. Puente S, Iñiguez A, Subirats M, Alonso MJ, Polo F, Moneo I. Gastroenteritis eosinofílica causada por sensibilización al polen.Med Clin (Barc) 1997; 108 (18): 698-700.

9. Martínez-Albadalejo M, Alguacil García G, González Pina B, De Paco M, Herranz T, Moreno-Requena J. Gastroenteritis eosinofílica. a propósito de un nuevo caso. An Med Interna (Madrid) 1997; 14 (4): 187-189.

10. Medina-Pérez M, Novales Velasco G, Medina Pérez A, Oliva Mompeán F. Gastroenteritis eosinofílica asociada con artritis reumatoide. Presentación como abdomen agudo. Rev Esp Enferm Dig. 1997; 89(2): 143-144.

11. Pérez-Millán A, martín-Lorente JL, López-Morante A, Yuguero L, Sáez Royuela F. Subserosal eosinophilic gastroenteritis treated efficaciously with sodium cromoglycate. Dig Dis Sci 1997; 42(2): 342-344.

12. Sánchez-Pobre P, López Ríos F, Colina F, Yela C, Manzano M, Rodríguez $\mathrm{S}$ et al. Colecistitis eosinofílica: una causa infrecuente de colecistectomía. Gastroenterol Hepatol 1997; 20 (1): 21-23.

13. Remacha B, Velicia R, del Villar A, Fernández P, Caro-Patón A. Enteritis eosinofílica como causa de obstrucción intestinal.Gastroenterol Hepatol 1999; 22: 352-355.

14. Borda F, Jiménez FJ, Martínez Peñuela JM, Echarri A, Martín-Granizo L y Aznarez R. Esofagitis eosinofílica: ¿Una entidad infradiagnostica-

da? Rev Esp Enf Ap Digest 1996; 88: 701-704

15. Talley NJ, Shorter RG, Phillips SF, Zinsmeister AR. Eosinophilic gastroenteritis: a clinicopathological study of patients with disease of the mucosa, muscle layer and subserosal tissues. Gut 1990; 31: 54-58.

16. Blackshaw AJ, Levinson DA. Eosinophilic infiltrates of the gastrointestinal tract. J Clin Pathol 1986; 39: 1-7.

17. Scudamore HH, Phillips SF, Swedlung HA. Food allergy manifested by eosinophilia, elevated immunoglobulin $\mathrm{E}$ level and protein-losing enteropathy: The syndrome of allergic gastroenteropathy. J Aller Clin Immunol 1982; 70: 129-134

18. Klein NC, Hargrove RL, Sleissenger MH, Jeffries GH. Eosinophilic gastroenteritis. Medicine (Baltimore) 1970; 49: 299-319.

19. Naylor AR. Eosinophilic gastroenteritis. Scott Med J 1990; 35: 163165

20. Fortman LM, Johansson JF, Baskin WW, Greenlaw RL. Eosinophilic ascitis without eosinophilia: a unique presentation of serosal eosinophilic gastroenteritis. Am J Gast 1993; 88: 1280-1281.

21. Matsushita MM, Hajiro K, Morita Y, Takakuwz H, Suzaki T. Eosinophilic gastroenteritis involving the entire digestive tract. Am J Gast 1995; 90: $1868-1870$.

22. Shweiki E, West JC, Klena JW, Kelley SE, Thompson A, Bross RJ, Tyler WB. Eosiniphilic gastroenteritis presenting as an obstructing cecal mass: a case report and review of the literature. Am J Gast 1999; 94: 3644-3645.

23. Vitellas KM, Bennet WF, Bova JG, Johnson JC, Greenson JK, Caldwell JH. Radiographic manifestations of eosinophilic gastroenteritis. Abdom Imaging 1995; 20: 406-413.

24. Mc Nabb PC, Flemming CR, Higgins JA. Transmural eosinophilic gastroenteritis with ascitis. Mayo Clin Proc 1979; 54: 119-122.

25. Winter HS, Madara JL, Stafford RJ. Functional and morphological assesment of acid reflux in children. Gastroenterology 1979; 78: 1923 1928.

26. Solís-Herruzo J, De Cuenca B, Muñoz-Yagüe MT. Laparoscopic findings in serosal eosinophilic gastroenteritis: report of two cases. Endoscopy $1988 ; 20: 152-155$

27. Rodríguez HE, Djohan RS, Cahill WJ. Laparoscopic diagnosis of eosinophilic gastroenteritis. J S Laparosc Surg 1998; 2: 181-184.

28. Mehammed Y, Feanny SJ, Shermann PM. Benefit of ketotifen in patients with eosinophilic gastroenteritis. Am J Med 1991; 90: 310-314

29. Di Gioacchino X, Pizzicanella G, Frenni W. Sodium cromoglycate in the treatment of eosinophilic gastroenteritis. Allergy 1990; $45: 161-166$. 